\title{
Pauli-Villars regularization of field theories on the light front
}

\author{
John HILLER* \\ University of Minnesota-Duluth, USA \\ E-mail: jhillered.umn.edu
}

Four-dimensional quantum field theories generally require regularization to be well defined. This can be done in various ways, but here we focus on Pauli-Villars (PV) regularization and apply it to nonperturbative calculations of bound states. The philosophy is to introduce enough PV fields to the Lagrangian to regulate the theory perturbatively, including preservation of symmetries, and assume that this is sufficient for the nonperturbative case. The numerical methods usually necessary for nonperturbative bound-state problems are then applied to a finite theory that has the original symmetries. The bound-state problem is formulated as a mass eigenvalue problem in terms of the light-front Hamiltonian. Applications to quantum electrodynamics are discussed.

Light Cone 2010 - LC2010

June 14-18, 2010

Valencia, Spain

\footnotetext{
*Speaker.
} 


\section{Introduction}

In order to solve a $(3+1)$-dimensional theory, the theory must be regulated in some way. In doing so, one should attempt to preserve as many symmetries as possible. We do this by adding enough Pauli-Villars (PV) [1] fields to regulate perturbation theory and assume that the nonperturbative eigenproblem is also regulated. Numerical methods are then applied to a finite theory, just as was the case for (1+1)-dimensional superrenormalizable theories [2]. From the Hamiltonian eigenproblem, we can compute wave functions as coefficients in Fock-state expansions, and from these, compute observables.

As a test of the approach, we consider QED. This is not meant to compete with perturbation theory; the numerical errors of the nonpertubative calculation are too large to resolve high-order contributions that perturbation theory computes directly. However, the method is intended for strong-coupling theories where perturbation theory is ineffective.

There have been a number of applications of the method. The first being an exploration in terms of a soluble, heavy-source model [3, 4]. The dressed-fermion state in Yukawa theory has been studied extensively [5, 6, 7], as have exact solutions in the limit of equal PV masses [8]. Applications to gauge theories have been primarily to the dressed-electron state in QED [9, 10, 11, 12, 13], but also to the photon eigenstate [14]. A scheme has been proposed for QCD [15].

In order to have a well-defined Fock-state expansion, the theories are quantized on the light front $[16,2]$. We define light-cone coordinates of time, $x^{+}=t+z$, and space, $\underline{x}=\left(x^{-}, \vec{x}_{\perp}\right)$, with $x^{-} \equiv t-z$ and $\vec{x}_{\perp}=(x, y)$. The light-cone energy is $p^{-}=E-p_{z}$ and momentum, $p=\left(p^{+}, \vec{p}_{\perp}\right)$, with $p^{+} \equiv E+p_{z}$ and $\vec{p}_{\perp}=\left(p_{x}, p_{y}\right)$. These lead to the mass-shell condition, $p^{2}=m^{2}$, in the form $p^{-}=\frac{m^{2}+p_{\perp}^{2}}{p^{+}}$.

We work with the standard parameterization, where the bare parameters of the Lagrangian are fixed by fits to physical constraints. There is the alternative of sector-dependent parameterization, where the bare parameters of the Lagrangian are allowed to depend on the Fock sector(s) on which the operators act. This was originally proposed by Perry, Harindranath, and Wilson [17] and applied to QED by Hiller and Brodsky [18]. More recent work with this approach has been by Karmanov, Mathiot, and Smirnov [19, 20]. Other nonperturbative approaches include lattice theory [21, 22], Dyson-Schwinger equations [23], effective-particle representations [24], and basis-function methods [25].

The remainder of this paper contains a brief description of light-front QED in Lorentz gauge, followed by discussion of the eigenproblem for the electron state in lowest truncation. The anomalous magnetic moment is calculated. We also compare the results of the sector-dependent approach, and then conclude with a brief summary.

\section{Light-front QED in Lorentz gauge}

The Lorentz-gauge QED Lagrangian, regulated by two PV fermion flavors and two PV photon flavors, is

$$
\mathscr{L}=\sum_{i=0}^{2}(-1)^{i}\left[-\frac{1}{4} F_{i}^{\mu v} F_{i, \mu \nu}+\frac{1}{2} \mu_{i}^{2} A_{i}^{\mu} A_{i \mu}-\frac{1}{2}\left(\partial^{\mu} A_{i \mu}\right)^{2}\right]
$$




$$
+\sum_{i=0}^{2}(-1)^{i} \bar{\psi}_{i}\left(i \gamma^{\mu} \partial_{\mu}-m_{i}\right) \psi_{i}-e_{0} \bar{\psi} \gamma^{\mu} \psi A_{\mu}
$$

where

$$
\psi=\sum_{i=0}^{2} \sqrt{\beta_{i}} \psi_{i}, A_{\mu}=\sum_{i=0}^{2} \sqrt{\xi_{i}} A_{i \mu}, \quad F_{i \mu \nu}=\partial_{\mu} A_{i v}-\partial_{v} A_{i \mu} .
$$

A subscript of $i=0$ indicates a physical field, and $i=1$ or 2 a PV field. The $i=1$ fields are chosen to have negative norm. The mass of the bare photon $\mu_{0}$ is zero.

The constants $\beta_{i}$ and $\xi_{i}$ control the coupling strengths of the various fields. We require that $\beta_{0}=1$ and $\xi_{0}=1$ and require the constraints $\sum_{i=0}^{2}(-1)^{i} \beta_{i}=0$ and $\sum_{i=0}^{2}(-1)^{i} \xi_{i}=0$. These guarantee the regularization and that the combinations $\psi$ and $A_{\mu}$ in (2.2) have zero norm. A third pair of constraints comes from requiring that the photon eigenstate have zero mass [14] and that the mass of the electron eigenstate becomes zero when $m_{0}$ is set to zero [10].

The nondynamical fermion fields satisfy the following constraints $(i=0,1,2)$ :

$$
i(-1)^{i} \partial_{-} \psi_{i-}+e_{0} A_{-} \sqrt{\beta_{i}} \psi_{-}=i \gamma^{0} \vec{\gamma}^{\perp} \cdot\left[(-1)^{i} \vec{\partial}_{\perp} \psi_{i+}-i e_{0} \sqrt{\beta_{i}} \vec{A}_{\perp} \psi_{+}\right]-(-1)^{i} m_{i} \gamma^{0} \psi_{i+} .
$$

From these we obtain a constraint on the null combination

$$
i \partial_{-} \psi_{-}=\left(i \gamma^{0} \vec{\gamma}^{\perp}\right) \cdot \vec{\partial}_{\perp} \psi_{+}-\gamma^{0} \sum_{i} m_{i} \sqrt{\beta_{i}} \psi_{i+} .
$$

The terms containing the photon field cancel because $\sum_{i}(-1)^{i} \beta_{i}=0$; therefore, light-cone gauge is not necessary. The nondynamical field $\psi_{-}$can then be constructed from a sum of $\psi_{i-}$ that satisfy the free-fermion constraint.

The mode expansion for the full Fermi field is

$$
\psi_{i}=\frac{1}{\sqrt{16 \pi^{3}}} \sum_{s} \int \frac{d \underline{k}}{\sqrt{k^{+}}}\left[b_{i s}(\underline{k}) e^{-i \underline{k} \cdot \underline{x}} u_{i s}(\underline{k})+d_{i,-s}^{\dagger}(\underline{k}) e^{i \underline{k} \cdot \underline{x}} v_{i s}(\underline{k})\right] .
$$

The spinors are defined in [26], and the nonzero anticommutators are $\left\{b_{i s}(\underline{k}), b_{i^{\prime} s^{\prime}}^{\dagger}\left(\underline{k}^{\prime}\right)\right\}=(-1)^{i} \delta_{i i^{\prime}} \delta_{s s^{\prime}} \delta\left(\underline{k}-\underline{k}^{\prime}\right)$ and $\left\{d_{i s}(\underline{k}), d_{i^{\prime} s^{\prime}}^{\dagger}\left(\underline{k}^{\prime}\right)\right\}=(-1)^{i} \delta_{i i^{\prime}} \delta_{s s^{\prime}} \delta\left(\underline{k}-\underline{k}^{\prime}\right)$.

The mode expansion for the $i$ th photon flavor is

$$
A_{i \mu}=\frac{1}{\sqrt{16 \pi^{3}}} \int \frac{d \underline{k}}{\sqrt{k^{+}}}\left[a_{i \mu}(\underline{k}) e^{-i \underline{k} \cdot \underline{x}}+a_{i \mu}^{\dagger}(\underline{k}) e^{i \underline{k} \cdot \underline{x}}\right],
$$

with the commutator $\left[a_{i \mu}(\underline{k}), a_{i^{\prime} v}^{\dagger}\left(\underline{k}^{\prime}\right)\right]=(-1)^{i} \delta_{i i^{\prime}} \varepsilon^{\mu} \delta_{\mu v} \delta\left(\underline{k}-\underline{k}^{\prime}\right)$. The metric signature is chosen to be $\varepsilon^{\mu}=(-1,1,1,1)$, for Gupta-Bleuler quantization [27]. Because we do not use light-cone gauge, there is no constraint on $A_{+}=A^{-}$, and, consequently, there will be no instantaneous photon interaction term [2] in the Hamiltonian.

We can now construct the light-front Hamiltonian $\mathscr{P}^{-}$from spinor matrix elements [14]:

$$
\begin{aligned}
\mathscr{P}^{-}= & \sum_{i, s} \int d \underline{p} \frac{m_{i}^{2}+p_{\perp}^{2}}{p^{+}}(-1)^{i} b_{i, s}^{\dagger}(\underline{p}) b_{i, s}(\underline{p})+\sum_{i, s} \int d \underline{p} \frac{m_{i}^{2}+p_{\perp}^{2}}{p^{+}}(-1)^{i} d_{i, s}^{\dagger}(\underline{p}) d_{i, s}(\underline{p}) \\
& +\sum_{l, \mu} \int d \underline{k} \frac{\mu_{l}^{2}+k_{\perp}^{2}}{k^{+}}(-1)^{l} \varepsilon^{\mu} a_{l \mu}^{\dagger}(\underline{k}) a_{l \mu}(\underline{k})
\end{aligned}
$$




$$
\begin{aligned}
& +\sum_{i, j, l, s, \mu} \sqrt{\beta_{i} \beta_{j} \xi_{l}} \int d \underline{p} d \underline{q}\left\{b _ { i , s } ^ { \dagger } ( \underline { p } ) \left[b_{j, s}(\underline{q}) V_{i j, 2 s}^{\mu}(\underline{p}, \underline{q})\right.\right. \\
& \left.+b_{j,-s}(\underline{q}) U_{i j,-2 s}^{\mu}(\underline{p}, \underline{q})\right] a_{l \mu}^{\dagger}(\underline{q}-\underline{p}) \\
& +b_{i, s}^{\dagger}(\underline{p})\left[d_{j, s}^{\dagger}(\underline{q}) \bar{V}_{i j, 2 s}^{\mu}(\underline{p}, \underline{q})+d_{j,-s}^{\dagger}(\underline{q}) \bar{U}_{i j,-2 s}^{\mu}(\underline{p}, \underline{q})\right] a_{l \mu}(\underline{q}+\underline{p}) \\
& \left.-d_{i, s}^{\dagger}(\underline{p})\left[d_{j, s}(\underline{q}) \tilde{V}_{i j, 2 s}^{\mu}(\underline{p}, \underline{q})+d_{j,-s}(\underline{q}) \tilde{U}_{i j,-2 s}^{\mu}(\underline{p}, \underline{q})\right] a_{l \mu}^{\dagger}(\underline{q}-\underline{p})+H . c .\right\} .
\end{aligned}
$$

The vertex functions can be found in [14]. The Hamiltonian does not contain any instantaneous fermion terms [2]. They cancel between physical and PV contributions because they are independent of the fermion mass and proportional to $(-1)^{i} \beta_{i}$ for the $i$ th flavor. The sum over flavors then yields $\sum_{i}(-1)^{i} \beta_{i}=0$. This is independent of the gauge choice.

\section{Electron eigenstate}

In the one-electron/one-photon truncation, the Fock-state expansion of the electron eigenstate, for total $J_{z}= \pm \frac{1}{2}$, is

$$
\left|\psi^{ \pm}(\underline{P})\right\rangle=\sum_{i} z_{i} b_{i \pm}^{\dagger}(\underline{P})|0\rangle+\sum_{i j s \mu} \int d \underline{k} C_{i j s}^{\mu \pm}(\underline{k}) b_{i s}^{\dagger}(\underline{P}-\underline{k}) a_{j \mu}^{\dagger}(\underline{k})|0\rangle .
$$

It is normalized according to $\left\langle\psi^{\sigma^{\prime}}\left(\underline{P}^{\prime}\right) \mid \psi^{\sigma}(\underline{P})\right\rangle=\delta\left(\underline{P}^{\prime}-\underline{P}\right) \delta_{\sigma^{\prime} \sigma}$. The second PV fermion flavor $(i=2)$ plays no role in this sector and can be removed. In order to have a positive norm, all physical quantities are computed from a projected state [9]:

$$
\begin{aligned}
\left|\psi_{\text {phys }}^{ \pm}(\underline{P})\right\rangle= & \sum_{i}(-1)^{i} z_{i} b_{0 \pm}^{\dagger}(\underline{P})|0\rangle+\sum_{s \mu} \int d \underline{k} \sum_{i=0}^{1} \sum_{j=0,2} \sqrt{\xi_{j}} \\
& \times \sum_{k=j / 2}^{j / 2+1} \frac{(-1)^{i+k}}{\sqrt{\xi_{k}}} C_{i k s}^{\mu \pm}(\underline{k}) b_{0 s}^{\dagger}(\underline{P}-\underline{k}) a_{j \mu}^{\dagger}(\underline{k})|0\rangle .
\end{aligned}
$$

To be an eigenstate of the light-cone Hamiltonian, the wave functions must satisfy the following coupled equations, with $y=k^{+} / P^{+}$:

$$
\begin{aligned}
{\left[M^{2}-m_{i}^{2}\right] z_{i}=} & \int\left(P^{+}\right)^{2} d y d^{2} k_{\perp} \sum_{j, l, \mu} \sqrt{\xi_{l}}(-1)^{j+l} \varepsilon^{\mu} \\
& \times\left[V_{j i \pm}^{\mu *}(\underline{P}-\underline{k}, \underline{P}) C_{j l \pm}^{\mu \pm}(\underline{k})+U_{j i \pm}^{\mu *}(\underline{P}-\underline{k}, \underline{P}) C_{j l \mp}^{\mu \pm}(\underline{k})\right],
\end{aligned}
$$

and

$$
\begin{aligned}
& {\left[M^{2}-\frac{m_{j}^{2}+k_{\perp}^{2}}{1-y}-\frac{\mu_{l}^{2}+k_{\perp}^{2}}{y}\right] C_{j l \pm}^{\mu \pm}(\underline{k})=\sqrt{\xi_{l}} \sum_{i^{\prime}}(-1)^{i^{\prime}} z_{i^{\prime}} P^{+} V_{j i^{\prime} \pm}^{\mu}(\underline{P}-\underline{k}, \underline{P}),} \\
& {\left[M^{2}-\frac{m_{j}^{2}+k_{\perp}^{2}}{1-y}-\frac{\mu_{l}^{2}+k_{\perp}^{2}}{y}\right] C_{j l \mp}^{\mu \pm}(\underline{k})=\sqrt{\xi_{l}} \sum_{i^{\prime}}(-1)^{i^{\prime}} z_{i^{\prime}} P^{+} U_{j i^{\prime} \pm}^{\mu}(\underline{P}-\underline{k}, \underline{P}) .}
\end{aligned}
$$

An index of $i$ corresponds to the one-electron sector and $j$ to the one-electron/one-photon sector. Therefore, in the sector-dependent approach, a mass $m_{i}$ in a vertex function is assigned the bare mass, and $m_{j}$ is the physical mass. In the standard parameterization, all are bare masses. 
The coupled equations can be solved analytically [9]. The wave functions $C_{i l s}^{\mu \pm}$ are

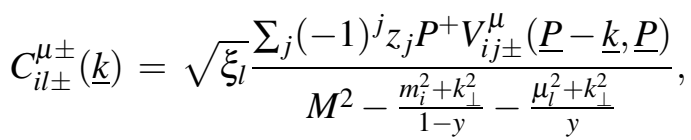

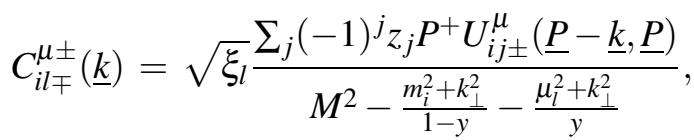

and the amplitudes satisfy

$$
\left(M^{2}-m_{i}^{2}\right) z_{i}=2 e_{0}^{2} \sum_{i^{\prime}}(-1)^{i^{\prime}} z_{i^{\prime}}\left[\bar{J}+m_{i} m_{i^{\prime}} \bar{I}_{0}-2\left(m_{i}+m_{i^{\prime}}\right) \bar{I}_{1}\right],
$$

with [9]

$$
\begin{aligned}
\bar{I}_{n}\left(M^{2}\right) & =\int \frac{d y d k_{\perp}^{2}}{16 \pi^{2}} \sum_{j l} \frac{(-1)^{j+l} \xi_{l}}{M^{2}-\frac{m_{j}^{2}+k_{\perp}^{2}}{1-y}-\frac{\mu_{l}^{2}+k_{\perp}^{2}}{y}} \frac{m_{j}^{n}}{y(1-y)^{n}}, \\
\bar{J}\left(M^{2}\right) & =\int \frac{d y d k_{\perp}^{2}}{16 \pi^{2}} \sum_{j l} \frac{(-1)^{j+l} \xi_{l}}{M^{2}-\frac{m_{j}^{2}+k_{\perp}^{2}}{1-y}-\frac{\mu_{l}^{2}+k_{\perp}^{2}}{y}} \frac{m_{j}^{2}+k_{\perp}^{2}}{y(1-y)^{2}} .
\end{aligned}
$$

There is also the identity [10] $\bar{J}=M^{2} \bar{I}_{0}$.

The analytic solution is [9]

$$
\alpha_{0 \pm}=\frac{\left(M \pm m_{0}\right)\left(M \pm m_{1}\right)}{8 \pi\left(m_{1}-m_{0}\right)\left(2 \bar{I}_{1} \pm M \bar{I}_{0}\right)}, z_{1}=\frac{M \pm m_{0}}{M \pm m_{1}} z_{0} .
$$

A graphical solution is given in [10]. In general, the lower sign yields the physical answer, because $m_{0}$ then becomes the physical mass $M=m_{e}$ at zero coupling. The coupling coefficient $\xi_{2}$ is fixed by requiring that $M=0$ when $m_{0}=0$. In this truncation, we can safely take the $m_{1} \rightarrow \infty$ limit, where $z_{1}=0, m_{1} z_{1} \rightarrow \pm\left(M-m_{0}\right) z_{0}$, and

$$
\alpha_{0 \pm}= \pm \frac{M\left(M \pm m_{0}\right)}{8 \pi\left(2 \bar{I}_{1} \pm M \bar{I}_{0}\right)}
$$

and the second PV photon flavor can be discarded. In the sector-dependent approach, $\bar{I}_{1}$ and $\bar{I}_{0}$ are independent of $m_{0}$, and the solution for $\alpha_{0}$ can be rearranged as an explicit expression for $m_{0}$

$$
m_{0}=\mp M+8 \pi \frac{\alpha_{0 \pm}}{M}\left(2 \bar{I}_{1} \pm M \bar{I}_{0}\right) .
$$

The anomalous magnetic moment is computed from the spin-flip matrix element of the electromagnetic current $J^{+}$[28]. In the one-photon truncation and in the limit where the PV electron mass $m_{1}$ is infinite, the expression for the anomalous moment is

$$
\begin{aligned}
a_{e}= & \frac{\alpha_{0}}{\pi} m_{e}^{2} z_{0}^{2} \int y^{2}(1-y) d y d k_{\perp}^{2} \\
& \times\left(\sum_{k=0}^{1} \frac{(-1)^{k}}{y m_{0}^{2}+(1-y) \mu_{k}^{2}+k_{\perp}^{2}-m_{e}^{2} y(1-y)}\right)^{2} .
\end{aligned}
$$

For the sector-dependent parameterization, the product $\alpha_{0} z_{0}^{2}$ is just $\alpha$, and the bare mass $m_{0}$ in the denominator is replaced by the physical mass $m_{e}$. Plots of the anomalous moment as a function of the regulator mass $\mu_{1}$ can be found in [12]. 


\section{Sector-dependent and standard parameterizations}

Even though we do not include the vacuum-polarization contribution to the dressed-electron state, the sector-dependent bare coupling is not equal to the physical coupling. Instead, they are related by $e_{0}=e / z_{0}$ [18], where $z_{0}$ is the amplitude for the bare-electron Fock state computed without projection onto the physical subspace. In general, the bare coupling would be $e_{0}=Z_{1} e / \sqrt{Z_{2 i} Z_{2 f} Z_{3}}$; this includes the truncation effect that splits the usual $Z_{2}$ into a product of different $\sqrt{Z_{2}}$ from each fermion leg [29]. With no fermion-antifermion loop, we have $Z_{3}=1$, and without a second photon, there is no vertex correction and $Z_{1}=1$. Also, only the fermion leg with no photon spectator will be corrected by $\sqrt{Z_{2}}$; therefore, we find $\sqrt{Z_{2 i} Z_{2 f}}=z_{0}$.

In the sector-dependent approach, the bare-electron amplitude without projection is determined, in the infinite- $m_{1}$ limit, by the normalization $1=z_{0}^{2}+e_{0}^{2} z_{0}^{2} \tilde{J}_{2}$, with

$$
\tilde{J}_{2}=\frac{1}{8 \pi^{2}} \int y d y d k_{\perp}^{2} \sum_{k=0}^{1}(-1)^{k} \frac{\left(y^{2}+2 y-2\right) m_{e}^{2}+k_{\perp}^{2}}{\left[k_{\perp}^{2}+(1-y) \mu_{k}^{2}+y^{2} m_{e}^{2}\right]^{2}} .
$$

Replacing $e_{0}$ by $e / z_{0}$, we can solve for $z_{0}$ as $z_{0}=\sqrt{1-e^{2} \tilde{J}_{2}}$ and find $e_{0}=e / \sqrt{1-e^{2} \tilde{J}_{2}}$. For large $\mu_{1}$, one finds $\tilde{J}_{2} \simeq \frac{1}{8 \pi^{2}}\left(\ln \frac{\mu_{1} \mu_{0}^{2}}{m_{e}^{3}}+\frac{9}{8}\right)$. Thus, $e_{0}$ can become imaginary and Fock-sector probabilities range outside $[0,1]$ due to IR and UV divergences, and consistency then imposes limits on $\mu_{0}$ and $\mu_{1}$ [12], as confirmed for Yukawa theory in [20].

In the standard parameterization, the bare amplitude is determined by $1=z_{0}^{2}+e^{2} z_{0}^{2} J_{2}$, with

$$
\begin{aligned}
J_{2}= & \frac{1}{8 \pi^{2}} \int y d y d k_{\perp}^{2}\left[m_{0}^{2}-4 m_{0} m_{e}(1-y)+m_{e}^{2}(1-y)^{2}+k_{\perp}^{2}\right] \\
& \times\left(\sum_{k=0}^{1}(-1)^{k} \frac{1}{\left[k_{\perp}^{2}+(1-y) \mu_{k}^{2}+y m_{0}^{2}-y(1-y) m_{e}^{2}\right]}\right)^{2} .
\end{aligned}
$$

Thus the bare amplitude is $z_{0}=1 / \sqrt{1+e^{2} J_{2}}$, which is driven to zero as $\mu_{1} \rightarrow \infty$ and causes most expectation values also to go to zero. Therefore, in this case there is a limit on $\mu_{1}$, but $\mu_{0}$ can be zero.

The anomalous moment in the sector-dependent case is

$$
\begin{aligned}
a_{e}= & \frac{\alpha}{\pi} m_{e}^{2} \int y^{2}(1-y) d y d k_{\perp}^{2} \\
& \sum_{k=0}^{1}(-1)^{k}\left(\frac{1}{y m_{e}^{2}+(1-y) \mu_{k}^{2}+k_{\perp}^{2}-m_{e}^{2} y(1-y)}\right)^{2}
\end{aligned}
$$

In the $\mu_{1} \rightarrow \infty, \mu_{0} \rightarrow 0$ limit, this becomes exactly the Schwinger result

$$
a_{e}=\frac{\alpha}{\pi} m_{e}^{2} \int \frac{d y d q_{\perp}^{2} /(1-y)}{\left[\frac{m_{e}^{2}+q_{\perp}^{2}}{1-y}+\frac{q_{\perp}^{2}}{y}-m_{e}^{2}\right]^{2}}=\frac{\alpha}{2 \pi} .
$$

However, this limit cannot be taken without making the underlying theory inconsistent. 


\section{Summary}

With use of PV regularization, one can formulate and solve nonperturbative problems in field theories. It is important to maintain symmetries, which can be done with additional PV fields, such as those introduced to maintain a zero photon mass and the chiral symmetry of the masslesselectron limit. It is best to regulate before applying numerical methods, to clearly separate limits of regulators from those of numerical convergence. The PV fields do add to the numerical load but also reduce it, by eliminating instantaneous fermion and instantaneous photon interactions.

For both the standard parameterization and the sector-dependent parameterization, truncation of the Fock space results in uncancelled divergences, which require that not all PV masses be taken to infinity; however, meaningful results can be extracted at finite PV masses. For the sectordependent approach, this is complicated by infrared divergences [12].

As discussed elsewhere [13], these methods have been extended to a truncation that includes two photons. The next step is to also include an electron-positron-pair contribution to the dressedelectron state and study charge renormalization as well as current covariance. This truncation will then include all contributions of order $\alpha^{2}$ to the anomalous moment. A calculation at large $\alpha$, where numerical errors in the order- $\alpha$ contribution would be small compared to the $\alpha^{2}$ contributions, could be compared with higher-order perturbation theory. It would also be interesting to consider the dressed electron in a magnetic field and extract its induced magnetic moment. In addition, as a precursor to consideration of mesons in QCD, one can compute two-fermion bound states in Yukawa theory and QED.

\section{Acknowledgments}

The work reported here was done in collaboration with S.S. Chabysheva and supported in part by the US Department of Energy and the Minnesota Supercomputing Institute.

\section{References}

[1] W. Pauli and F. Villars, Rev. Mod. Phys. 21 (1949) 434.

[2] For reviews of light-cone quantization, see M. Burkardt, Adv. Nucl. Phys. 23, 1 (2002); S.J. Brodsky, H.-C. Pauli, and S.S. Pinsky, Phys. Rep. 301 (1998) 299.

[3] S.J. Brodsky, J.R. Hiller, and G. McCartor, Phys. Rev. D 58 (1998) 025005.

[4] S.J. Brodsky, J.R. Hiller, and G. McCartor, Phys. Rev. D 60 (1999) 054506.

[5] S.J. Brodsky, J.R. Hiller, and G. McCartor, Phys. Rev. D 64 (2001) 114023.

[6] S.J. Brodsky, J.R. Hiller, and G. McCartor, Ann. Phys. 305 (2003) 266.

[7] S.J. Brodsky, J.R. Hiller, and G. McCartor, Ann. Phys. 321 (2006) 1240.

[8] S.J. Brodsky, J.R. Hiller, and G. McCartor, Ann. Phys. 296 (2002) 406.

[9] S.J. Brodsky, V.A. Franke, J.R. Hiller, G. McCartor, S.A. Paston, and E.V. Prokhvatilov, Nucl. Phys. B 703 (2004) 333.

[10] S.S. Chabysheva and J.R. Hiller, Phys. Rev. D 79 (2009) 114017. 
[11] S.S. Chabysheva, A nonperturbative calculation of the electron's anomalous magnetic moment, Ph.D. thesis, Southern Methodist University ProQuest Dissertations \& Theses 33690092009.

[12] S.S. Chabysheva and J.R. Hiller, Ann. Phys. 325 (2010) 2435.

[13] S.S. Chabysheva and J.R. Hiller, Phys. Rev. D 81 (2010) 074030.

[14] S.S. Chabysheva and J.R. Hiller, Phys. Rev. D 82 (2010) 034004.

[15] S.A. Paston and V.A. Franke, Theor. Math. Phys. 112 (1997) 1117 [Teor. Mat. Fiz. 112 (1997) 399]; S.A. Paston, V.A. Franke, and E.V. Prokhvatilov, Theor. Math. Phys. 120 (1999) 1164 [Teor. Mat. Fiz. 120 (1999) 417].

[16] P.A.M. Dirac, Rev. Mod. Phys. 21 (1949) 392.

[17] R.J. Perry, A. Harindranath, and K.G. Wilson, Phys. Rev. Lett. 65 (1990) 2959; R.J. Perry and A. Harindranath, Phys. Rev. D 43 (1991) 4051.

[18] J.R. Hiller and S.J. Brodsky, Phys. Rev. D 59 (1998) 016006.

[19] V. A. Karmanov, J. F. Mathiot, and A. V. Smirnov, Phys. Rev. D 77 (2008) 085028.

[20] V. A. Karmanov, J. F. Mathiot, and A. V. Smirnov, arXiv:1006.5640 [hep-th].

[21] For reviews of lattice theory, see M. Creutz, L. Jacobs and C. Rebbi, Phys. Rep. 95 (1983) 201; J.B. Kogut, Rev. Mod. Phys. 55 (1983) 775; I. Montvay, ibid. 59 (1987) 263; A.S. Kronfeld and P.B. Mackenzie, Ann. Rev. Nucl. Part. Sci. 43 (1993) 793; J.W. Negele, Nucl. Phys. A553 (1993) 47c; K.G. Wilson, Nucl. Phys. B (Proc. Suppl.) 140 (2005) 3; J.M. Zanotti, PoS (LAT2008) 007. For recent discussions of meson properties and charm physics, see for example C. McNeile and C. Michael [UKQCD Collaboration], Phys. Rev. D 74 (2006) 014508; I. Allison et al. [HPQCD Collaboration], Phys. Rev. D 78 (2008) 054513.

[22] M. Burkardt and S. Dalley, Prog. Part. Nucl. Phys. 48 (2002) 317 and references therein; S. Dalley and B. van de Sande, Phys. Rev. D 67 (2003) 114507; D. Chakrabarti, A.K. De, and A. Harindranath, Phys. Rev. D 67 (2003) 076004; M. Harada and S. Pinsky, Phys. Lett. B 567 (2003) 277; S. Dalley and B. van de Sande, Phys. Rev. Lett. 95 (2005) 162001; J. Bratt, S. Dalley, B. van de Sande, and E. M. Watson, Phys. Rev. D 70 (2004) 114502. For work on a complete light-cone lattice, see C. Destri and H.J. de Vega, Nucl. Phys. B290 (1987) 363; D. Mustaki, Phys. Rev. D 38 (1988) 1260.

[23] C.D. Roberts and A.G. Williams, Prog. Part. Nucl. Phys. 33 (1994) 477; P. Maris and C.D. Roberts, Int. J. Mod. Phys. E12 (2003) 297; P.C. Tandy, Nucl. Phys. B (Proc. Suppl.) 141 (2005) 9.

[24] S. D. Glazek and R. J. Perry, Phys. Rev. D 78 (2008) 045011; S.D. Głazek and J. Mlynik, Phys. Rev. D 74 (2006) 105015; S.D. Głazek, Phys. Rev. D 69 (2004) 065002; S.D. Głazek and J. Mlynik, Phys. Rev. D 67 (2003) 045001; S.D. Głazek and M. Wieckowski, Phys. Rev. D 66 (2002) 016001.

[25] J.P. Vary et al., Phys. Rev. C 81 (2010) 035205.

[26] G.P. Lepage and S.J. Brodsky, Phys. Rev. D 22 (1980) 2157.

[27] S.N. Gupta, Proc. Phys. Soc. (London) A63 (1950) 681; K. Bleuler, Helv. Phys. Acta 23 (1950) 567.

[28] S.J. Brodsky and S.D. Drell, Phys. Rev. D 22 (1980) 2236.

[29] D. Mustaki, S. Pinsky, J. Shigemitsu, and K. Wilson, Phys. Rev. D 43 (1991) 3411. 